\title{
NAC-loaded electrospun scaffolding system with dual compartments for the osteogenesis of rBMSCs in vitro
}

This article was published in the following Dove Press journal: International Journal of Nanomedicine

\author{
Yuanjing Zhu',* \\ Fangfang Songl,* \\ Yanyun Ju ${ }^{2}$ \\ Liyuan Huang' \\ Lu Zhang' \\ Chuliang Tang' \\ Hongye Yang' \\ Cui Huang'
}

'Center for Smart Materials and Devices, State Key Laboratory of Advanced Technology for Materials Synthesis and Processing, School of Materials Science and Engineering, Wuhan University of Technology, Wuhan, Hubei, China; ${ }^{2}$ Center for Smart Materials and Devices, State Key Laboratory of Advanced Technology for Materials Synthesis and Processing, Wuhan University of Technology, Wuhan, Hubei, China

*These authors contributed equally to this work
Correspondence: Cui Huang

The State Key Laboratory Breeding Base of Basic Science of Stomatology (Hubei-MOST) and Key Laboratory of Oral Biomedicine Ministry of Education (KLOBM), School and Hospital of Stomatology, Wuhan University, Luoyu Road 237, Hongshan District, Wuhan 430079, Hubei, China

Tel +86278764 6337

Email huangcui@whu.edu.cn
Purpose: In this study, we aimed to develop a unique $N$-acetyl cysteine (NAC)-loaded polylactic-co-glycolic acid (PLGA) electrospun system with separate compartments for the promotion of osteogenesis.

Materials and methods: We first prepared solutions of NAC-loaded mesoporous silica nanoparticles (MSNs), PLGA, and NAC in $N, N$-dimethylformamide and tetrahydrofuran for the construction of the electrospun system. We then fed solutions to a specific injector for electrospinning. The physical and chemical properties of the scaffold were characterized through scanning electron microscopy, transmission electron microscopy, and Fourier transform infrared spectroscopy. The release of NAC and Si from different PLGA scaffolds was estimated. The cell viability, cell growth, and osteogenic potential of rat bone marrow-derived stroma cell (rBMSCs) on different PLGA scaffolds were evaluated through MTT assay, live/dead staining, phalloidin staining, and Alizarin red staining. The expression levels of osteogenic-related markers were analyzed through real-time PCR (qRT-PCR).

Results: NAC was successfully loaded into MSNs. The addition of MSNs and NAC decreased the diameters of the electrospun fibers, increased the hydrophilicity and mechanical property of the PLGA scaffold. The release kinetic curve indicated that NAC was released from $(\mathrm{PLGA}+\mathrm{NAC}) /(\mathrm{NAC} @ \mathrm{MSN})$ in a biphasic pattern, that featured an initial burst release stage and a later sustained release stage. This release pattern of NAC encapsulated on the (PLGA + NAC)/(NAC@MSN) scaffolds enabled to prolong the high concentrations of release of NAC, thus drastically affecting the osteogenic differentiation of rBMSCs.

Conclusion: A PLGA electrospun scaffold was developed, and MSNs were used as separate nanocarriers for recharging NAC concentration, demonstrating the promising use of (PLGA + $\mathrm{NAC}) /(\mathrm{NAC} @ \mathrm{MSN})$ for bone tissue engineering.

Keywords: bone tissue engineering, $N$-acetyl cysteine, osteogenesis, electrospun, mesoporous silica nanoparticles, drug compartment

\section{Introduction}

Bone defects are common but serious clinical problems that are caused by trauma, inflammation, or cancer. Bone tissue engineering has been developed as a promising technique for the treatment of bone defects. Tissue-engineered bone scaffolds solve several problems including limited bone supply and the risks of additional surgery and donor-site morbidity, caused by autogenous bone graft. ${ }^{1-3}$ Qualified scaffolds should possess optimal physical properties, mimic the basic properties of bone extracellular matrix (ECM), and promote osteogenesis by providing additional bioactive molecules. ${ }^{4,5}$ 
Numerous small molecules regulate the differentiation and proliferation of stem, precursor, and terminally differentiated cells. ${ }^{6} \mathrm{~N}$-acetyl cysteine (NAC) is a water-soluble and membrane-permeable small molecule with various intra- and extracellular functionalities, such as antioxidant potential, ability to enhance cytocompatibility and enhance osteogenic differentiation..$^{7-9} \mathrm{NAC}$ is cheaper, safer, and easier to synthesize and deliver than other growth factors, such as BMP-2, and lacks any dose-dependent side effects. ${ }^{10} \mathrm{~A}$ scaffolding system that comprises NAC loaded on a collagenous sponge scaffold has been shown to promote bone regeneration in vitro and in vivo by accelerating osteogenesis. ${ }^{9}$ In this scaffolding system, however, NAC is simply absorbed onto a collagen sponge scaffold. This loading approach may result in the burst release of NAC. In turn, this release pattern limits the biological and clinical utility of the scaffolds.

Polylactic-co-glycolic acid (PLGA), an FDA-approved polymer, has been widely used in bone tissue engineering and in controlled release devices for the deliver therapeutically bioactive agents because of its assured biocompatibility and controllable biodegradation. ${ }^{11-13}$ PLGA undergoes hydrolysis upon degradation. The degradation products of PLGA include biologically compatible and metabolizable moieties, such as glycolic acid and lactic acid. These acidic degradation products can be removed through the tricarboxylic acid cycle and do not affect the normal cell because of their slow excretion rates. ${ }^{14}$ Nevertheless, hydrophobic structure and mechanical properties of PLGA limit its application for bone regeneration. ${ }^{15,16}$ Furthermore, although Kim et al ${ }^{17}$ showed that bioactive molecules can be easily incorporated into PLGA fibers through blending, their release cannot be controlled and instead occurs in a single intense burst. One solution to these problems is the incorporation of inorganic particles, such as mesoporous silica nanoparticles (MSNs), into PLGA scaffolds. MSNs are biocompatible nanocarriers with high surface areas and tunable particle/pore sizes. ${ }^{18,19}$ Given these features, MSNs can serve as molecular anchors for the loading of bioactive agents. MSNs can slowly and stably release loaded agents. The elastic modulus and hardness of PLGA scaffolds have been increased through the addition of MSNs. ${ }^{20}$ Furthermore, $\mathrm{Si}$ ions released from MSNs promote the osteogenic differentiation of hMSCs. ${ }^{21}$

In our study, we constructed an electrospun scaffold system that can release NAC to promote osteogenesis. In this system, the small bioactive molecule NAC is encapsulated into MSNs, which are immobilized in biodegradable NACcontaining PLGA fibers through the electrospinning technique. The fabricated scaffold system is designated as (PLGA +NAC)/(NAC@MSN) (Figure 1A and B). ${ }^{22}$ It simultaneously acts as a cell carrier and a controlled drug release vehicle. PLGA is electrospun with MSNs into fibers that are architecturally similar to natural collagen fibers and possess the physical characteristics of the ECM which contains organic phases and inorganic minerals. ${ }^{23,24}$ Dual compartments control the release of NAC from the (PLGA+NAC)/(NAC@MSN) system. rBMSCs are used to evaluate the osteogenic potential of the (PLGA + NAC)/(NAC@MSN) electrospun scaffold. We believe that this drug delivery system would maximize the osteogenic ability of rBMSCs over the long term.
A

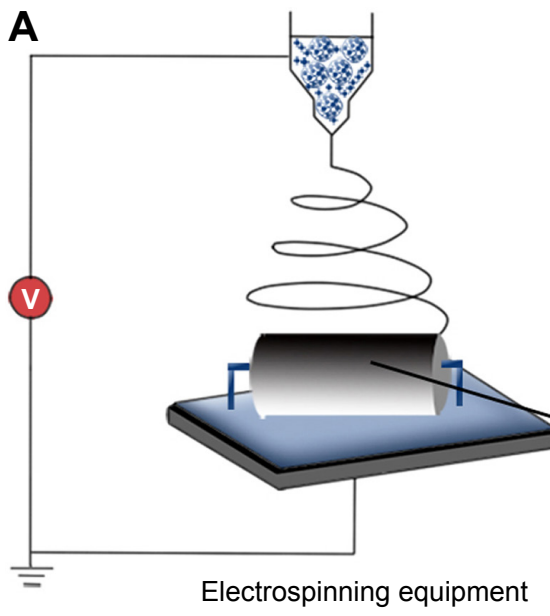

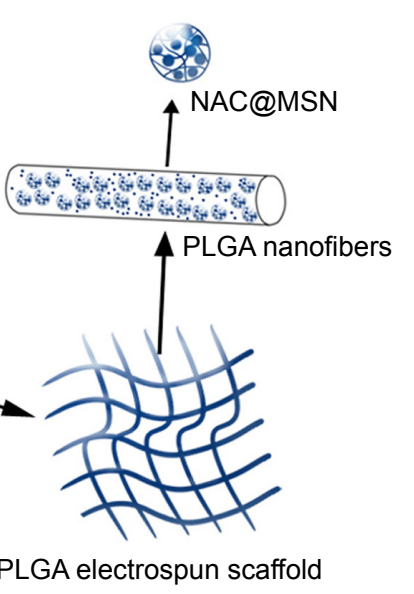

B

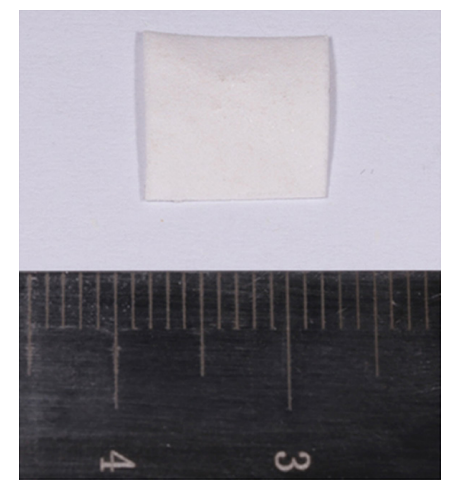

Figure I Fabrication of the NAC-loaded PLGA electrospun scaffold and PNNM electrospun scaffold.

Notes: Fabrication of the PLGA electrospun scaffold (A) showing fiber formation. Fibers are organized on the rotor receiver of the equipment. Gross view of the PNNM electrospun scaffold (B) NAC@MSN particles and NAC are embedded in the fibers. Reprinted from Acta Biomater, 8(5), Song B, Wu C, Chang J, Dual drug release from electrospun poly(lactic-co-glycolic acid)/mesoporous silica nanoparticles composite mats with distinct release profiles, 190I-1907, Copyright (20I2), with permission from Elsevier. ${ }^{22}$ Abbreviations: NAC, N-acetyl cysteine; PLGA, polylactic-co-glycolic acid; MSNs, mesoporous silica nanoparticles; PNNM, PLGA with free NAC and NAC@MSN. 


\section{Materials and methods \\ Reagents}

PLGA (MW =150,000, lactic acid: glycolic acid =90:10) was supplied by Jinan Daigang Biotechnology Co., Ltd. (Jinan, Shandong, China). Tetraethyl orthosilicate (TEOS) and cetyltrimethylammonium bromide (CTAB) were purchased from Aladdin Biochem Technology Co., Ltd. (Shanghai, China). Alizarin Red S, Sodium hydroxide, ethyl alcohol, tetrahydrofuran (THF), and $N, N$-dimethylformamide (DMF) were obtained from Sinopharm Chemical Reagent (Shanghai, China). Minimum essential medium Eagle-alpha modification ( $\alpha$-MEM), penicillin-streptomycin, and trypsin were procured from Hyclone (South Logan, UT, USA). Fetal bovine serum (FBS) was purchased from Gibco (Grand Island, NY, USA). Prime Script RT reagent Kit (DRR037A) and SYBR ${ }^{\circledR}$ Green PCR Master Mix (DRR420A) were provided by TaKaRa (Tokyo, Japan). NAC, fluorescein diacetate, propidium iodide (PI), 4',6-diamidino-2phenylindole (DAPI), and phalloidin were obtained from Sigma-Aldrich Co. (St Louis, MO, USA). Dimethyl sulfoxide (DMSO) and the cell viability reagent MTT were purchased from BioFroxx Co. (Einhausen, Germany).

\section{Fabrication of the drug delivery system} MSNs were synthesized as follows. First, $1 \mathrm{~g}$ of CTAB and $3.5 \mathrm{~mL}$ of $\mathrm{NaOH}(2 \mathrm{M})$ were added to $480 \mathrm{~mL}$ of distilled water. Second, the solution was heated to $80^{\circ} \mathrm{C}$. After 4 hours of stirring, $5 \mathrm{~mL}$ of TEOS was added dropwise to the solution. After $\sim 2$ hours of reaction, the sample was washed with distilled water and ethanol and then collected through centrifugation at 10,000 rpm. The surfactant was removed through three extraction cycles in a mixture of $4 \mathrm{~mL}$ of $\mathrm{HCl}$ $(37 \% \mathrm{w} / \mathrm{v})$ and $200 \mathrm{~mL}$ of ethanol at $90^{\circ} \mathrm{C}$.

In loading NAC onto MSNs, $1.0 \mathrm{~g}$ of MSNs was immersed in $100 \mathrm{~mL}$ of NAC solution $(10 \mathrm{mg} / \mathrm{mL})$. After 48 hours of stirring in the dark, NAC-loaded MSNs (NAC@ MSN) were collected through centrifugation and then freezedried for 12 hours. The amount of NAC loaded onto MSNs was measured through thermogravimetric analysis (TGA). The drug-loading rate was calculated with the following equation: loading efficiency $=($ loaded MSNs - initial MSNs initial MSN) $\times 100$.

The electrospun (PLGA + NAC)/(NAC@MSN) scaffolds (PNNM scaffolds) containing NAC@MSN (20\%w/v, relative to PLGA) and NAC (5\% w/v relative to PLGA) were prepared through the following procedures. First, $0.7 \mathrm{~g}$ of MSNs was added to a mixture containing $7.5 \mathrm{~mL}$ of DMF and $2.5 \mathrm{~mL}$ of THF. After dispersion, $3.5 \mathrm{~g}$ of PLGA and
$0.21 \mathrm{~g}$ of NAC were added to the suspension. The suspension was stirred until it becomes homogeneous. PLGA suspensions containing only NAC $(5 \% \mathrm{w} / \mathrm{v}$, relative to PLGA, PN scaffold) or only NAC@MSN (20\% w/v relative to PLGA, PNM scaffold) were also prepared. The pure PLGA scaffold was subsequently used as the control group. Electrospinning experiments were performed at room temperature under the relative humidity of $\sim 50 \%$. The applied voltage was controlled at $20 \mathrm{kV}$. The distance between the tip of the needle and the collector was $15 \mathrm{~cm}$. The feeding rate of the suspension was fixed at $2 \mathrm{~mL} / \mathrm{h}$ and controlled by a syringe pump. All of the groups of electrospun scaffolds were vacuum-dried for 48 hours to remove the residual solvent.

\section{Characterization of the MSN, NAC@ MSN, and PNNM scaffold}

The size, morphological characteristics, and distribution of MSNs were determined through scanning electron microscopy (SEM) (Zeiss, Göttingen, Germany) and transmission electron microscopy (TEM) (JEM-2100, JEOL, Tokyo, Japan). The drug-loading rate and chemical contents of NAC@MSN were confirmed through TGA (TA Instruments, New Castle, DE, USA) and Fourier transform infrared spectroscopy (FTIR, Thermo Fisher Scientific Inc, Waltham, MA, USA). The morphological characteristics and structure of all electrospun scaffolds were characterized through SEM and TEM. The average fiber diameter was quantified on the basis of SEM images by using ImageJ. A total of 90 fibers were randomly selected from each image $(\times 5,000$ magnification) for analysis. The swelling ratios, contact angles, and mechanical properties of the scaffolds were determined. For swelling analysis, dried scaffolds were immersed for 24 hours in PBS solution at $37^{\circ} \mathrm{C}$ in a shaking water bath $(50 \mathrm{rpm})$. All of the groups of scaffolds were soaked in $5 \mathrm{~mL}$ distilled deionized water for degradation analysis at $37^{\circ} \mathrm{C}$ in a shaking water bath $(100 \mathrm{rpm})$ for 21 days, and the degradation of the scaffolds was observed through SEM. ${ }^{25}$

The nanoindentation hardness and elastic modulus were tested by a Hysitron TI950 TriboIndenter (Hysitron Inc., Minneapolis, MN, USA) system and were calculated using the Oliver and Pharr method. ${ }^{26}$ Before the tests, the system was calibrated using quartz samples. A Hysitron spherical diamond probe with $5.28 \mu \mathrm{m}$ tip radius was used. The feedback of indenter is set to be "displacement control", and the maximum indentation depth was $4,000 \mathrm{~nm}$. A 2 seconds hold time at maximum load and 10 seconds at loading and unloading time were applied. 


\section{Drug-release kinetics of Si and NAC}

Cumulative Si release from the scaffolds was measured at 6 , 12,18 , and 24 days. PN, PNM, and PNNM scaffold samples $(20 \mathrm{mg}$ ) were incubated in $10 \mathrm{~mL}$ of distilled deionized water. The Si concentrations of each group were quantified through inductively coupled plasma-atomic emission spectroscopy (ICP-AES, Thermo Fisher Scientific Inc). Cumulative NAC release was detected through ultraviolet spectrophotometry at $24,72,120,144,168,216$, and 264 hours. PNNM, PNM, and PN scaffold samples (20 mg) were immersed in $2 \mathrm{~mL}$ of distilled deionized water. Then, the samples were mixed with 4-chloro-7-nitrobenzofurazan ( $0.006 \mathrm{wt} \%)$ chromogenic agent at the ratio of $1: 18$. After 30 minutes of reaction, NAC concentrations were calculated on the basis of the absorbance of the solution at $423 \mathrm{~nm}$.

\section{Biocompatibility and osteo-promotive effects of PNNM scaffolds in vitro}

Cell culture rBMSCs were extracted from the bone marrow of 4-week-old Sprague Dawley rats. All animal procedures were approved by the Institutional Ethical Board of Wuhan University and in accordance with the guidelines for the Care and Use of Laboratory Animals of Wuhan University. Primary cells were culture in $\alpha$-MEM containing $10 \%$ FBS and $1 \%$ streptomycin-penicillin and maintained under 5\% $\mathrm{CO}_{2}$ atmosphere at $37^{\circ} \mathrm{C}$. The rBMSCs were passaged upon reaching $70 \%-80 \%$ confluence and used at passage 3 or 4 for further studies. The medium was changed every 3 days.

\section{Cell proliferation, morphology, and adhesion}

Before the test was performed, the samples of pure PLGA, PN, PNM, or PNNM electrospun scaffolds were cut into $0.5 \times 0.5 \mathrm{~cm}$ mats and sterilized under UV for 2 hours per side. rBMSCs were seeded onto the scaffolds in 96-well plates at a density of $5 \times 10^{3}$ cells per well. The cells were initially cultured for 48 hours in growth culture medium. The culture medium in each well was then replaced with $200 \mu \mathrm{L}$ of MTT $(5 \mathrm{mg} / \mathrm{mL})$, and the cells were cultured for 2 hours. The MTT solution in each well was replaced with $100 \mu \mathrm{L}$ of DMSO. The absorbance of the solution was measured at $490 \mathrm{~nm}$.

The electrospun fibers were cut into $1 \times 1 \mathrm{~cm}$ mats, placed into 24-well plates, and sterilized. The rBMSCs were seeded at a density of $2 \times 10^{4}$ cells per mat. In the live/dead staining assay, the cells on the scaffolds were stained with fluorescein diacetate $(1 \mu \mathrm{g} / \mathrm{mL})$ and PI $(1 \mu \mathrm{g} / \mathrm{mL})$ and then visualized under confocal laser scanning microscopy (CLSM) (Olympus, Japan). Live cells were stained green by fluorescein diacetate, and dead cells were stained red by PI. In phalloidin staining, cells were initially incubated with $4 \%$ paraformaldehyde for 10 minutes. The actin cytoskeleton was dyed with phalloidin $(5 \mu \mathrm{g} / \mathrm{mL})$ for 30 minutes in the dark. Next, nuclei were stained with DAPI $(0.1 \mu \mathrm{g} / \mathrm{mL})$ for 2 minutes. The cells were observed and imaged through CLSM.

\section{qRT-PCR}

Samples were cut into $2 \times 2 \mathrm{~cm}$ mats and transferred to sixwell plates. rBMSCs were seeded onto scaffold samples at a density of $1 \times 10^{6}$ per well. After 7 days of culture, the scaffolds were disposed in TRIzol, and total RNA was collected for qRT-PCR analysis. Total RNA was reverse transcribed into cDNA with a Prime Script RT Reagent kit, and cDNA was used as qRT-PCR template. The qRTPCR primers used in this experiment are shown in Table 1. GAPDH mRNA levels were used as an internal control for the normalization of mRNA levels.

\section{ECM mineralization}

Each scaffold was cut into $1 \times 1 \mathrm{~cm}$ mats and placed in transwell plates. rBMSCs were seeded at a density of $2 \times 10^{5}$ per well in 24-well plates and cultured for 14 days in a growth medium. Then, the scaffolds were removed, and the cells were washed with PBS and immersed in 4\% paraformaldehyde for 10 minutes. The cells were stained with Alizarin red $(40 \mathrm{mM})$ in distilled water $(\mathrm{pH} 8.3)$ for 30 minutes at $37^{\circ} \mathrm{C}$, washed gently with distilled water, and observed. For quantitative analysis, the mineralization nodules were dissolved in $500 \mu \mathrm{L}$ of $10 \%$ cetylpyridinium chloride in

Table I Primer sequences for qRT-PCR

\begin{tabular}{l|l|l}
\hline Gene & Forward primer & Reverse primer \\
\hline rat-GAPDH & 5'-CACAGTCAAGGCTGAGAATG-3' $^{\prime}$ & $5^{\prime}$-GGTGGTGAAGACGCCAGTA-3' \\
\hline rat-ALP & 5'-CGTCTCCATGGTGGATTATGCT-3' $^{\prime}$ & $5^{\prime}$-CCCAGGCACAGTGGTCAAG-3' \\
\hline rat-Runx2 & $5^{\prime}$-TCTTCCCAAAGCCAGAGCG-3' & $5^{\prime}$-TGCCATTCGAGGTGGTCG-3' \\
\hline rat-OCN & $5^{\prime}$-GCCCTGACTGCATTCTGCCTCT-3' & $5^{\prime}$-TCACCACCTTACTGCCCTCCTG-3' \\
\hline
\end{tabular}


sodium phosphate $(10 \mathrm{mM})$, and absorbance was measured at $620 \mathrm{~nm}$.

\section{Statistical analysis}

In vitro experiments were independently performed with three replicate measurements. Data were presented as mean \pm SD. One-way ANOVA was conducted to determine significant differences among groups. A $P$-value of $<0.05$ was considered significant.

\section{Results}

Morphological characteristics and physical properties of MSNs and NAC@MSN

SEM and TEM observations reveal that the spherical MSNs have highly uniform sizes and morphologies (Figure 2A and B). Extensive pore structures can be observed on the surfaces of MSNs (Figure 2B). The particle size distribution of MSNs was quantified on the basis of SEM images by using ImageJ. The average diameter of MSNs is $69 \mathrm{~nm}$ (Figure 2C).
A

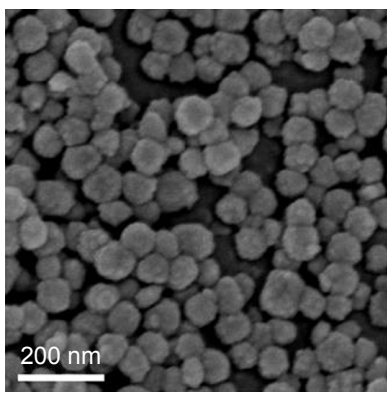

B

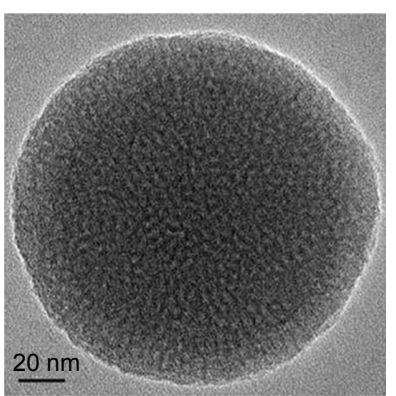

C

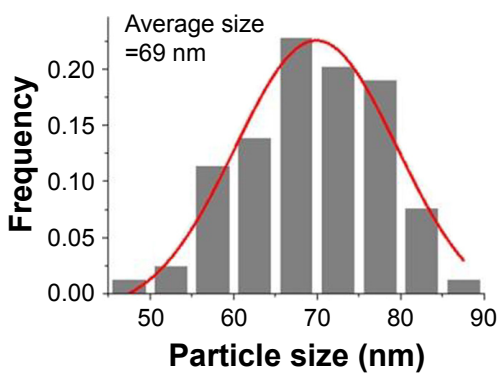

D

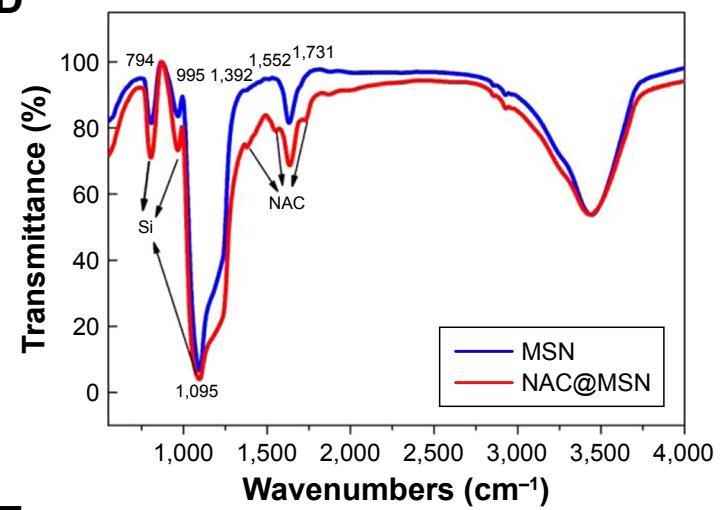

E

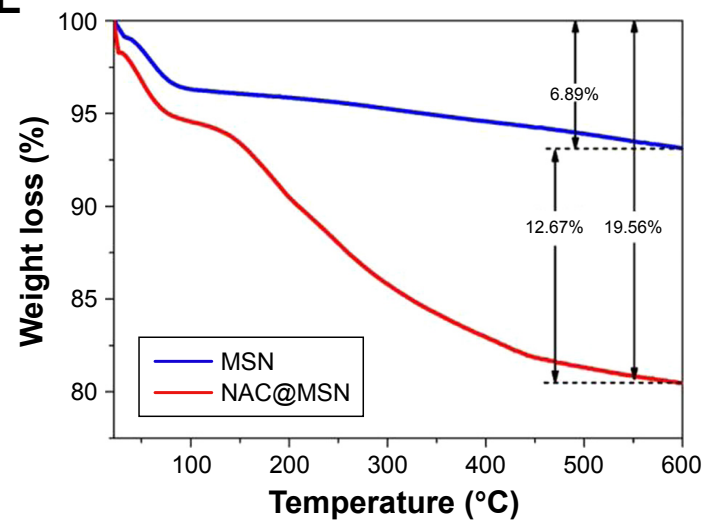

F

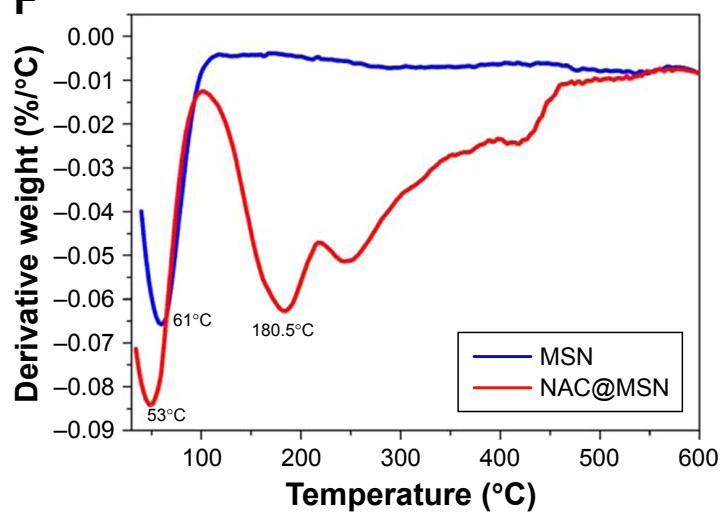

Figure 2 SEM image, TEM image, and particle size distribution of MSNs, and chemical analyses of MSNs after loading with NAC.

Notes: SEM image of MSNs (A). TEM image of MSNs (B). Particle size distribution of MSNs (C). Infrared spectra of MSNs and NAC@MSN (D). Arrows indicate the characteristic peaks of Si at 794, 995, and I,095 $\mathrm{cm}^{-1}$, and the characteristic peaks of NAC at I,392, I,552, and I,73I cm ${ }^{-1}$ (D). Thermogravimetric analysis of MSNs before and after loading with NAC (E). Derivative thermogravimetric analysis of MSNs before and after loading with NAC (F).

Abbreviations: SEM, scanning electron microscope; TEM, transmission electron microscopy; MSNs, mesoporous silica nanoparticles; NAC, N-acetyl cysteine; NAC@MSN, NAC-loaded MSN. 
The FTIR spectra of MSNs exhibit peaks at 995 and $794 \mathrm{~cm}^{-1}$, which are characteristic of asymmetric and symmetric $\mathrm{Si}-\mathrm{O}$ stretching vibrations. The peak at $1,095 \mathrm{~cm}^{-1}$ represents the $\mathrm{Si}-\mathrm{O}-\mathrm{Si}$ antisymmetric stretching vibration. ${ }^{27}$ The peak at $1,731 \mathrm{~cm}^{-1}$ corresponds to the stretching vibrations of $\mathrm{C}=\mathrm{O}$ bonds, and the peaks at 1,552 and $1,392 \mathrm{~cm}^{-1}$ correspond to amide bands (Figure 2D). All of these small peaks are the characteristic peaks of NAC..$^{28}$

The amount of NAC absorbed on MSNs was studied through TGA. The TGA thermograms of MSNs and NAC@MSN show two weight loss stages (Figure 2E). The first stage occurs at around $100^{\circ} \mathrm{C}$, which is mainly attributed to the evaporation of adsorbed water. The second stage takes place at $100^{\circ} \mathrm{C}$ to $600^{\circ} \mathrm{C}$ and is caused by the elimination of organic matter, particularly, NAC (Figure 2F). The weight losses of MSNs and NAC@MSN are 6.89 and $19.56 \mathrm{wt} \%$, respectively, suggesting that a high amount of NAC, $\sim 12.67 \mathrm{wt} \%$, is loaded onto MSN.

\section{Morphological characteristics and physical properties of MSNs and NAC@MSN}

The average thickness of the scaffold for cell studies is $0.3 \pm 0.05 \mathrm{~mm}$. The morphological characteristics and diameter distribution of the electrospun scaffolds are shown in SEM images. Pure PLGA fibers have the largest average diameter, which is $1,475 \mathrm{~nm}$. The average diameters of PN, PNM, and PNNM fibers are 823, 937, and $696 \mathrm{~nm}$, respectively (Figure $3 \mathrm{~A}_{4}-\mathrm{D}_{4}$ ). The surfaces of the pure PLGA and PN fibers appear smooth (Figure $3 \mathrm{~A}_{1}, \mathrm{~A}_{2}, \mathrm{~B}_{1}$, and $\mathrm{B}_{2}$ ), and the fibers of PNM and PNNM appear rough because of the presence of additional MSNs (Figure $3 \mathrm{C}_{1}, \mathrm{C}_{2}, \mathrm{D}_{1}$, and $\mathrm{D}_{2}$ ). Bead-like structures are present on the surfaces of PNM and PNNM fibers. The TEM images (Figure $3 \mathrm{~A}_{3}-\mathrm{D}_{3}$ ) show that MSNs are homogeneously encapsulated within the electrospun PLGA fibers (Figure $3 \mathrm{C}_{3}-\mathrm{D}_{3}$ ). The surfaces of the fibers roughen with the extrusion of some particles.

The morphological characteristics of the four groups of the electrospun scaffolds after 21 days of degradation are shown in Figure $3 \mathrm{~A}_{5}-\mathrm{D}_{5}$. All of the scaffolds become swollen after they are immersed in deionized water for 21 days. More pores are formed on the surface of PNM and PNNM fibers after incubation (Figure $3 \mathrm{C}_{5}-\mathrm{D}_{5}$ ), which may be caused by the loss of MSNs located on the surface of fibers. In comparison with PNN and PNNM scaffolds, the PLGA and PN fibers are fragmented, and some fibers are fused with one another (Figure 3A-B).

Contact angle analysis was performed to determine the surface wettability and hydrophilicity of the fibers. Contact angles of $<90^{\circ}$ are indicative of high wettability, and they favor the diffusion of liquid on the materials. By contrast, contact angles of more than $90^{\circ}$ correspond to low wettability. ${ }^{29}$ As shown in Figure 4A the contact angle value of the PNNM scaffold is lower than that of other scaffolds. In Figure 4B, the swelling ratios of all of the scaffolds are not significantly different. Figure $4 \mathrm{C}$ and D shows the average nanoindentation hardness and elastic modulus of pure PLGA, PN, PNM, and PNNM scaffolds. Both hardness and elastic modulus are higher in PN, PNM, and PNNM scaffold compared to the pure PLGA scaffold. Moreover, the PNNM scaffold displayed the highest harness and elastic modulus, followed by PNM, PN, and pure PLGA group in order. However, there is no significant difference in harness between PN and PNM scaffold.

\section{Kinetics of Si and NAC release}

NAC concentration was quantified through UV spectrophotometry. Figure 5A shows the release profile of NAC from PN, PNM, and PNNM scaffolds. Both PN and PNNM groups display an initial burst release, and NAC becomes exhausted within the first 4 days in the PN group. By contrast, the NAC release from the PNNM group exhibits a biphasic pattern characterized by an initial burst-release stage during the first 4 days and a long-term, gradual, and continuous release stage at which high NAC concentrations are sufficiently maintained to promote cell differentiation. Even though the PNM scaffold releases NAC continuously, it fails to release NAC at levels sufficient for cell stimulation.

Si concentration was quantified through ICP-AES. Figure 5B shows that $\mathrm{Si}$ ions are gradually and continuously released over a long period. This release pattern is suggestive of MSNs degradation.

\section{In vitro cellular study}

Cell viability rBMSCs were seeded on the scaffolds to investigate the influence of the scaffolds on cell viability. The viability of rBMSCs in various groups was tested through the MTT assay. In Figure 6A, cell activity is significantly higher in the PN, PNM, and PNNM groups than in the control group. This result suggests that the addition of NAC and MSNs positively affects cell viability. In line with the MTT results, the live/dead staining results of all the four groups reveal the almost complete absence of dead cells (red) and indicate that all of the prepared scaffolds are biocompatible (Figure 6 $\mathrm{B}_{1}-\mathrm{E}_{1}$ ).

\section{Cell adhesion and morphology}

rBMSCs were stained with phalloidin and DAPI at 48 hours after seeding (Figure $6 \mathrm{~B}_{2}-\mathrm{E}_{2}$ ) to visualize cell morphology 

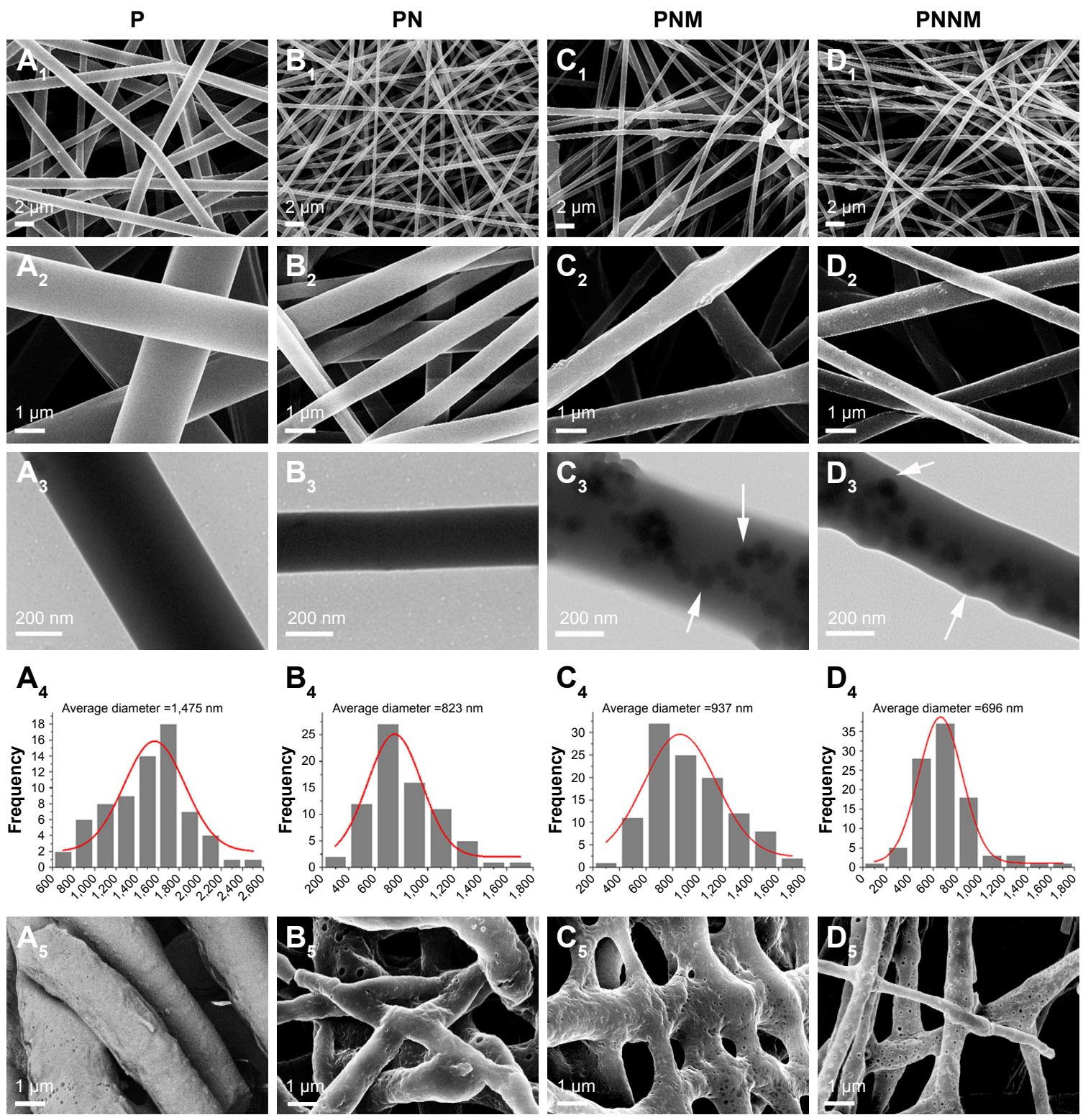

Figure 3 SEM image, TEM image, fiber diameter distribution, and degradation of pure PLGA, PN, PNM, and PNNM scaffolds.

Notes: SEM images, TEM images, fiber diameter distribution, and degradation after 21 days of pure PLGA scaffolds $\left(\mathbf{A}_{1}-\mathbf{A}_{5}\right)$, PN scaffolds $\left(\mathbf{B}_{1}-\mathbf{B}_{5}\right)$, PNM scaffolds $\left(\mathbf{C}_{1}-\mathbf{C}_{5}\right)$, and PNNM scaffolds $\left(\mathbf{D}_{1}-\mathbf{D}_{5}\right)$. The arrows point to MSNs $\left(\mathbf{C}_{3}\right)$ and NAC@MSN $\left(\mathbf{D}_{3}\right)$.

Abbreviations: SEM, scanning electron microscope; TEM, transmission electron microscopy; PLGA, polylactic-co-glycolic acid; PN, PLGA with free NAC; PNM, PLGA with NAC@MSN; PNNM, PLGA with free NAC and NAC@MSN; MSNs, mesoporous silica nanoparticles; NAC, N-acetyl cysteine; NAC@MSN, NAC-loaded MSN.

and adhesion. CLSM results show that the cells can attach and spread on all of the PLGA scaffolds. The cells exhibit clearly defined filopodia, lamellipodia, and polygonal shapes. No apparent differences are found among the four groups.

\section{qRT-PCR}

After 7 days of induction, the mRNA expression levels of ALP and Runx2 in the PN and PNNM groups are higher than those in the pure PLGA and PNM groups (Figure 7A and B). By contrast, their mRNA expression levels in the pure PLGA and PNM groups are not significantly different.
The mRNA expression levels of ALP in the PN and PNNM groups are 1.9- and 1.6-fold higher than those in the pure PLGA group, respectively. The expression levels of Runx2 in the PN and PNNM groups are 1.4- and 1.5-fold higher than those in the pure PLGA group, respectively. The mRNA expression levels of OCN (Figure 7C) in the PNNM scaffolds are the highest among the four groups. The expression levels of OCN in the PNM and PNNM scaffolds are 2.2- and 5.1-fold higher than those in the pure PLGA, respectively. The expression pattern of OCN may be attributed to the sustained release of NAC that enables the continuous induction of osteogenesis. 

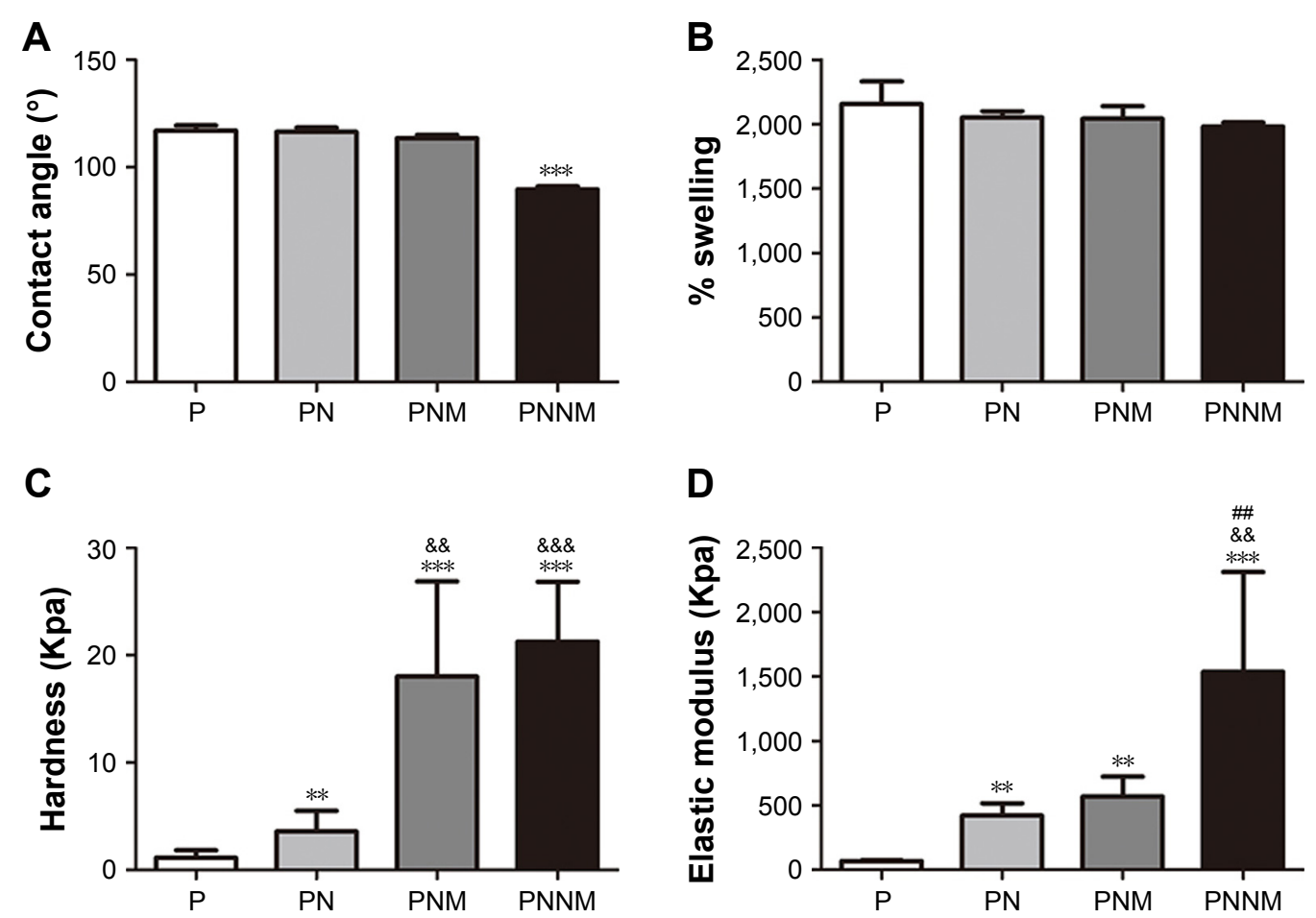

Figure 4 Water contact angle, swelling rate, nanoindentation hardness, and elastic modulus of pure PLGA, PN, PNM, and PNNM scaffolds.

Notes: Water contact angle of pure PLGA, PN, PNM, and PNNM scaffolds (A). Swelling rate of pure PLGA, PN, PNM, and PNNM scaffolds (B). Nanoindentation hardness and elastic modulus of pure PLGA, PN, PNM, and PNNM scaffolds (C and $\mathbf{D})$ ). ** represents for $P<0.01$ and *** represents $P<0.00 \mathrm{I}$ relative to the pure PLGA group (A); \&\& represents $P<0.01$ and ${ }^{\text {\&\&\& }}$ represents $P<0.00$ I relative to the $P N$ group; ${ }^{\#}$ represents $P<0.01$ relative to the PNM group.

Abbreviations: PLGA, polylactic-co-glycolic acid; PN, PLGA with free NAC; NAC, N-acetyl cysteine; PNM, PLGA with NAC@MSN; NAC@MSN, NAC-loaded MSNs; MSNs, mesoporous silica nanoparticles; PNNM, PLGA with free NAC and NAC@MSN.

\section{Alizarin red staining}

Mineralization nodules were detected through Alizarin red staining after 14 days of induction. In Figure 7D, the largest area of nodule mineralization is observed in the PNNM group. By contrast, minimal nodule mineralization is found in the three other groups. This result is supported by semiquantitative analysis findings (Figure 7E) possibly because of the extended maintenance of NAC concentrations at functional levels through the two-stage release of NAC from PNNM scaffolds.
A

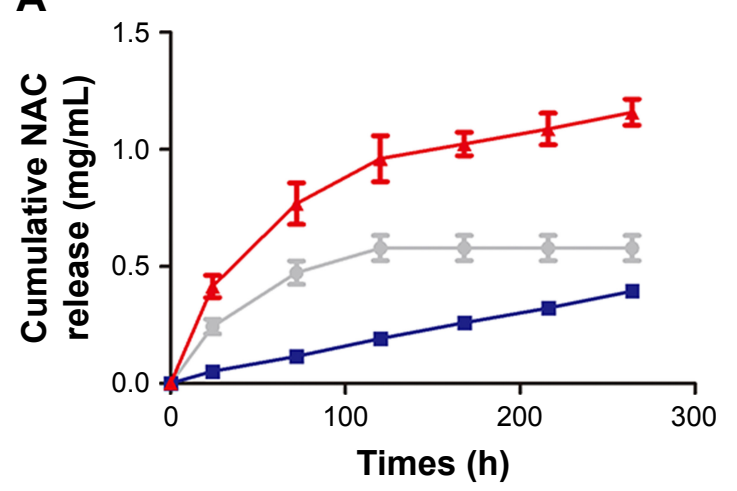

B

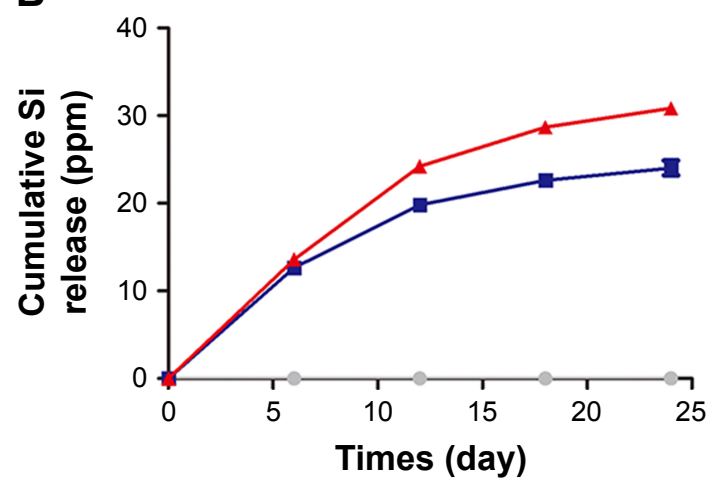

Figure 5 Drug-release kinetics of Si and NAC.

Notes: NAC release profiles of PN, PNM, and PNNM scaffolds during 0-280 hours in distilled water solution (A). Cumulative Si release profiles of PN, PNM, and PNNM scaffolds after 24 days in distilled water solution (B).

Abbreviations: PN, PLGA with free NAC; PNM, PLGA with NAC@MSN; PNNM, PLGA with free NAC and NAC@MSN; NAC, N-acetyl cysteine; NAC@MSN, NACloaded MSN. 
A

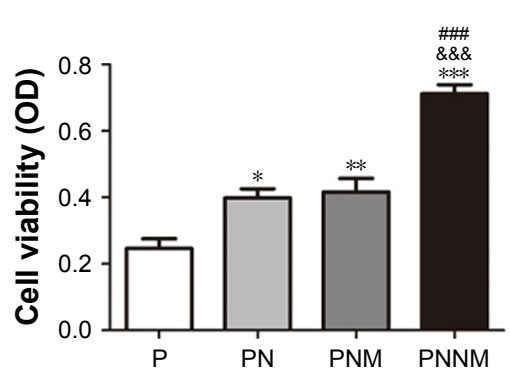

$P$
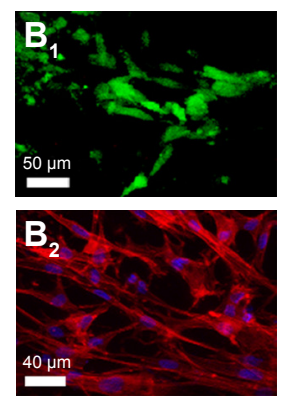

PN
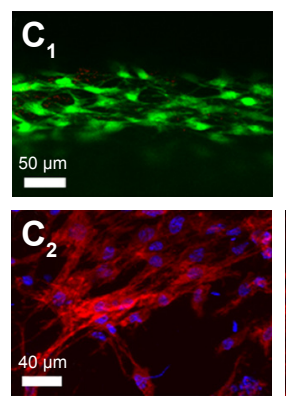

PNM
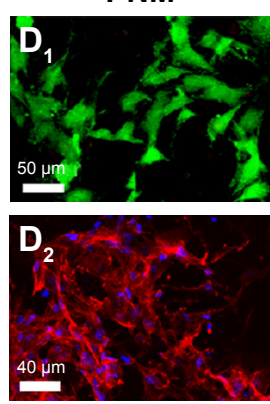

PNNM

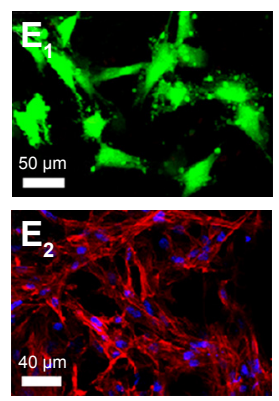

Figure 6 Cell viability and CLSM images of rBMSCs seeded on pure PLGA, PN, PNM, and PNNM scaffolds. CLSM images of shape of osteoblasts cultured under different conditions.

Notes: Cell viability values of rBMSCs $\left(\mathbf{A}_{)}\right.$cultured on samples for 48 hours; Live/dead staining of rBMSCs $\left(\mathbf{B}_{1}-\mathbf{E}_{1}\right)$ and actin cytoskeleton of $r B M S C_{s}\left(\mathbf{B}_{2}-\mathbf{E}_{2}\right)$ examined by CLSM at 48 hours on Pure PLGA $\left(\mathbf{B}_{1}, \mathbf{B}_{2}\right), \mathrm{PN}\left(\mathbf{C}_{1}, \mathbf{C}_{2}\right)$, PNM $\left(\mathbf{D}_{1}, \mathbf{D}_{2}\right)$, and PNNM $\left(\mathbf{E}_{1}, \mathbf{E}_{2}\right)$ sample. * represents $P<0.05$, ** represents $P<0.01$, and $* * *$ represents $P<0.001$ relative to the pure PLGA group (A). Green color indicates live rBMSCs in Figure $6 \mathbf{B}_{1}-\mathbf{E}_{1}$, red color indicates cytoskeleton of rBMSCs in $\mathbf{B}_{2}-\mathbf{E}_{2}$, and the blue color indicates cell nucleus in $\mathbf{B}_{2}-\mathbf{E}_{2}$. ${ }^{\text {s }}$ \& represents $P<0.001$ relative to the $P N$ group;

Abbreviations: CLSM, confocal laser scanning microscopy; rBMSCs, rat bone marrow stromal cells; PN, PLGA with free NAC; PNM, PLGA with NAC@MSN; PNNM, PLGA with free NAC and NAC@MSN; PLGA, polylactic-co-glycolic acid; MSNs, mesoporous silica nanoparticles; NAC, N-acetyl cysteine; NAC@MSN, NAC-loaded MSN.

\section{Discussion}

In the present study, an electrospun scaffold system is constructed to deliver NAC (Figure 1A), which comprises two separate compartments for NAC storage. In our system, NAC is encapsulated into MSNs nanocarriers, which are further electrospun with NAC-containing PLGA fibers. In previous studies, NAC is an antioxidative and anti-inflammatory drug that is widely used in various therapeutic strategies. ${ }^{30-32}$
A

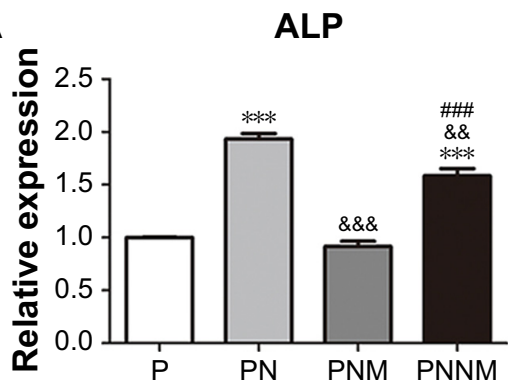

B

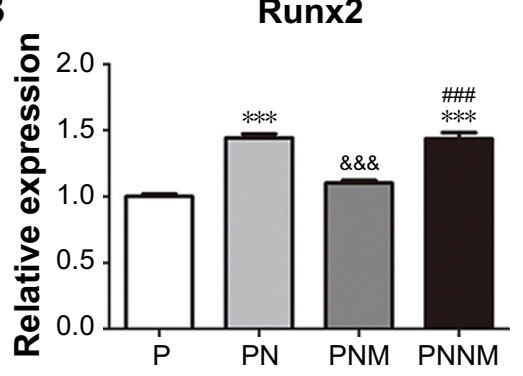

C

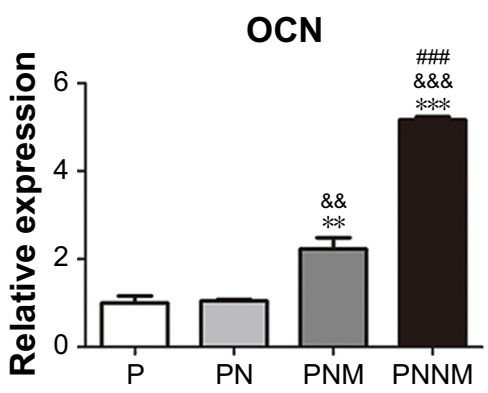

D
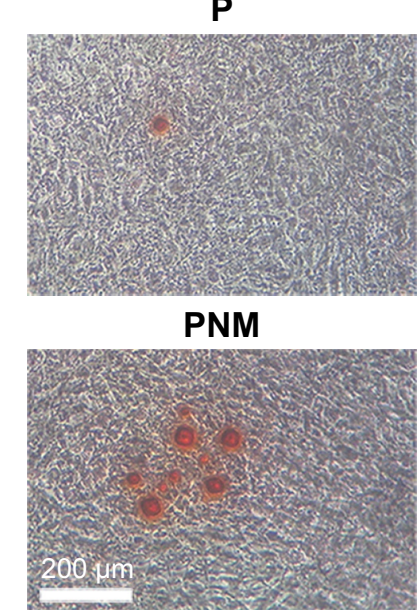

PN

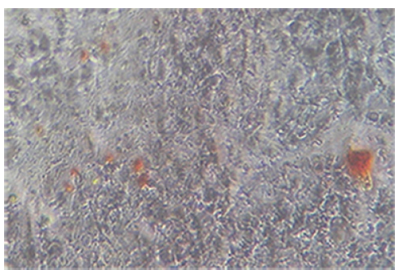

PNNM

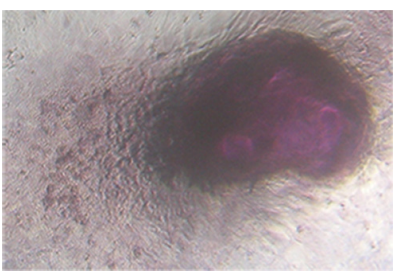

E

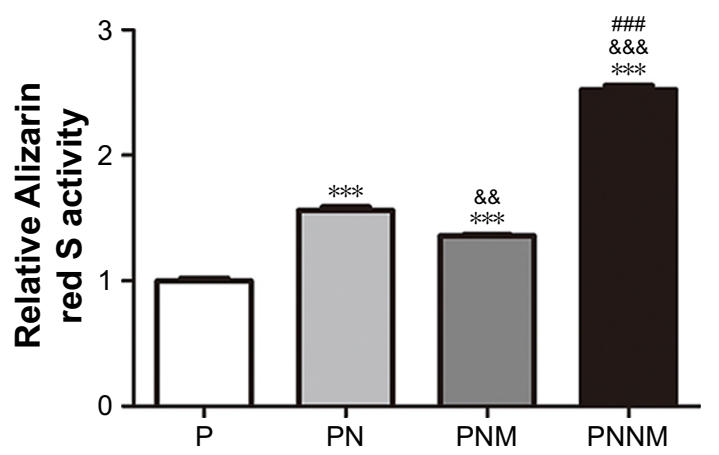

Figure 7 ALP, Runx2, and OCN expression, Alizarin red staining and relative qualification of Alizarin red.

Notes: ALP (A), Runx2 (B), and OCN (C) expression of rBMSCs seeded on pure PLGA, PN, PNM, and PNNM scaffolds for 7 days; Alizarin red staining (D) and relative qualification of Alizarin red (E) of rBMSCs cultured for 14 days with pure PLGA, PN, PNM, and PNNM scaffolds; ** represents $P<0.01$ and $* * *$ represents $P<0.00 \mathrm{I}$ relative to the pure PLGA group; \&\& represents $P<0.0$ I, \&\&\& represents $P<0.00$ I relative to the PN group; represents $P<0.001$ relative to the $P N N M$.

Abbreviations: ALP, alkaline phosphatase; OCN, osteocalcin; rBMSCs, rat bone marrow stromal cells; PN, PLGA with free NAC; PNM, PLGA with NAC@MSN; PNNM, PLGA with free NAC and NAC@MSN; PLGA, polylactic-co-glycolic acid; MSN, mesoporous silica nanoparticles; NAC, N-acetyl cysteine; NAC@MSN, NAC-loaded MSN. 
Yamada et al $1^{9,33}$ reported that NAC also exhibits osteogenic and angiogenetic properties, that is, NAC can promote osteogenesis when it is loaded on collagen scaffolds; however, these scaffolds cannot be used for the long-term sustainable release of NAC.

Electrospun scaffolds exhibit good characteristics for applications in drug delivery and bone repair application. ${ }^{34}$ Electrospinning technology can produce PLGA fibers with specific diameters and organizations. The interconnected structures of electrospun PLGA fibers mimic the size range of the bone ECM collagen fibrous component. MSNs are used as a reservoir to recharge NAC upon degradation. The addition of MSNs into PLGA electrospun fibers can construct two compartments for NAC loading, which enables the sustainable long-term release of NAC.

The addition of NAC and MSNs decrease the contact angle of the material surface through synergy between NAC, which contains amine and carboxylic functional groups, and MSN, which contains silanol groups. The addition of NAC and NAC@MSN can narrow fiber diameters. In general, the incorporation of MSNs and NAC into the polymer may increase the conductivity and viscosity of PLGA fibers, which exert opposing effects on fiber diameter. This result is consistent with the results of Mehrasa et al, ${ }^{35}$ who obtained thinner fibers by adding MSNs to PLGA polymers. PLGA polymer undergoes hydrolytic degradation and biodegradation in an aqueous environment by cleaving its backbone linkages. The results suggest that addition of NAC and MSNs can stabilize the PLGA fibers upon degradation. Furthermore, the addition of NAC and MSNs could increase the hardness and elastic modulus of PLGA fibers. One explanation may be that the addition of NAC and MSNs may strengthen the interaction between hydroxyl groups of MSNs, nitrogenous groups of NAC and carbonyl groups of PLGA through enhancing more hydrogen bond formation, which may result in higher mechanical property and lower degradation of PNNM and PNM. ${ }^{36}$

The release of NAC from the hierarchical PNNM scaffold in vitro is divided into the early burst stage and the later sustained and gradual release stage. At the early stage of release, NAC concentration drastically increases as NAC rapidly diffuses from the PLGA scaffold into the medium because of the direct physical integration of molecules within scaffold results in a matrix-type drug release characterized by a burst release rate. ${ }^{37}$ At the later release stage, the encapsulated NAC should first be initially dissociated from the MSNs to the PLGA matrix and then be subsequently released from the PLGA fibers to the outer solution. These results demonstrate that the combination of the separate compartments of MSNs nanocarriers and PLGA fibers, that is, the combination of two different dissociation pathways, can slow down the diffusion rate of NAC, thereby controlling NAC release and achieving a successive release pattern.

rBMSCs, important seed cells in bone tissue engineering, are used to evaluate the bioactivity of the hierarchical PNNM scaffolds. Cell viability tests reveal that all of the four groups enable cell growth. The PNNM group exhibits the highest cell activity. This result indicates that NAC and MSNs positively affect cell viability. The osteogenic potential of the scaffolds is consistent with the release curves of NAC. ALP and Runx 2 are early markers of osteogenesis, whereas OCN is a later marker of osteogenesis. ${ }^{38}$ qRT-PCR results show that ALP and Runx2 expression levels in the PN and PNNM groups are higher than those in the pure PLGA and PNM groups. One possible explanation for this result is that only the burst release of NAC from the PLGA fibers can provide the effective NAC concentration required at the early stage of osteogenesis. The expression levels of the later marker OCN are higher in the PNM and PNNM groups than in the pure PLGA and PN groups because the persistent recharge effect of NAC from MSNs in PNM and PNNM scaffolds promotes the continuous osteogenic differentiation of rBMSCs. On the contrary, the burst release of NAC from the PN group lasts four early days. PNNM coordinates two features in one system: the initial burst release of NAC promotes an initiate osteogenic differentiation at the early stage and the sustained release of NAC provides sustained osteogenic stimulation for a long time. The results of mineralized nodule formation and relative Alizarin red activity show that the PNNM group provides the most intense osteogenic stimuli among the four electrospun scaffold groups after 14 days' culture.

Therefore, our developed drug delivery system with dual drug release modes is a favorable system for the delivery of the bioactive molecule NAC in the treatment of bone defects and bone tissue engineering. In addition, NAC also possesses other excellent properties, such as antimicrobial, angiogenic, and anti-inflammatory activities. ${ }^{7,30,32}$ With these characteristics, PNNM may have other potential clinical applications, such as the treatment of inflammatory bone defects and mass bone defects through vascularization, the improvement of metabolism- or aged-associated bone decline. Further research is necessary to evaluate the potential applications of the NACloaded hierarchical PNNM scaffold developed in this work.

\section{Conclusion}

We developed an NAC-loaded electrospun scaffold system with dual compartments in which NAC is embedded into 
MSNs nanoparticles and then encapsulated in PLGA fibers premixed with NAC. Our PNNM system enables the sustained and controlled release of NAC and is a favorable rBMSC cell carrier. Overall, the PNNM scaffold system shows great potential for promoting osteogenic differentiation of rBMSCs, although in vivo studies need to be performed in the future.

\section{Acknowledgment}

This work was supported by the National Natural Science Foundation of China (81571012 and 81771112) and the fundamental Research Funds for the Central University (413000042 and 413000060).

\section{Author contributions}

All authors contributed to data analysis, drafting and revising the article, gave final approval of the version to be published, and agree to be accountable for all aspects of the work.

\section{Disclosure}

The authors report no conflicts of interest in this work.

\section{References}

1. Lee SS, Huang BJ, Kaltz SR, et al. Bone regeneration with low dose BMP-2 amplified by biomimetic supramolecular nanofibers within collagen scaffolds. Biomaterials. 2013;34(2):452-459.

2. O'Keefe RJ, Mao J. Bone tissue engineering and regeneration: from discovery to the clinic-an overview. Tissue Eng Part B Rev. 2011;17(6): 389-392.

3. Salgado AJ, Coutinho OP, Reis RL. Bone tissue engineering: state of the art and future trends. Macromol Biosci. 2004;4(8):743-765.

4. Boccaccini AR, Blaker JJ. Bioactive composite materials for tissue engineering scaffolds. Expert Rev Med Devices. 2005;2(3):303-317.

5. Collignon AM, Lesieur J, Vacher C, Chaussain C, Rochefort GY Strategies developed to induce, direct, and potentiate bone healing. Front Physiol. 2017;8:927.

6. Xu Y, Shi Y, Ding S. A chemical approach to stem-cell biology and regenerative medicine. Nature. 2008;453(7193):338-344.

7. Oikawa S, Yamada K, Yamashita N, Tada-Oikawa S, Kawanishi S. $\mathrm{N}$-acetylcysteine, a cancer chemopreventive agent, causes oxidative damage to cellular and isolated DNA. Carcinogenesis. 1999;20(8): $1485-1490$.

8. Zhu Y, Gu YX, Mo JJ, et al. N-acetyl cysteine protects human oral keratinocytes from Bis-GMA-induced apoptosis and cell cycle arrest by inhibiting reactive oxygen species-mediated mitochondrial dysfunction and the PI3K/Akt pathway. Toxicol In vitro. 2015;29(8): 2089-2101.

9. Yamada M, Tsukimura N, Ikeda T, et al. N-acetyl cysteine as an osteogenesis-enhancing molecule for bone regeneration. Biomaterials. 2013;34(26):6147-6156.

10. James AW, Lachaud G, Shen J, et al. A review of the clinical side effects of bone morphogenetic Protein-2. Tissue Eng Part B Rev. 2016;22(4): 284-297.

11. Kapoor DN, Bhatia A, Kaur R, Sharma R, Kaur G, Dhawan S. PLGA: a unique polymer for drug delivery. Ther Deliv. 2015;6(1):41-58.

12. Gentile P, Chiono V, Carmagnola I, Hatton PV. An overview of poly(lactic-co-glycolic) acid (PLGA)-based biomaterials for bone tissue engineering. Int J Mol Sci. 2014;15(3):3640-3659.
13. Li D, Sun H, Jiang L, et al. Enhanced biocompatibility of PLGA nanofibers with gelatin/nano-hydroxyapatite bone biomimetics incorporation. ACS Appl Mater Interfaces. 2014;6(12):9402-9410.

14. Panyam J, Labhasetwar V. Biodegradable nanoparticles for drug and gene delivery to cells and tissue. Adv Drug Deliv Rev. 2003;55(3): 329-347.

15. Félix Lanao RP, Jonker AM, Wolke JG, Jansen JA, van Hest JC, Leeuwenburgh SC. Physicochemical properties and applications of poly(lactic-co-glycolic acid) for use in bone regeneration. Tissue Eng Part B Rev. 2013;19(4):380-390.

16. Pan Z, Ding J. Poly(lactide-co-glycolide) porous scaffolds for tissue engineering and regenerative medicine. Interface Focus. 2012;2(3): 366-377.

17. Kim K, Luu YK, Chang C, et al. Incorporation and controlled release of a hydrophilic antibiotic using poly(lactide-co-glycolide)-based electrospun nanofibrous scaffolds. J Control Release. 2004;98(1):47-56.

18. Song Y, Li Y, Xu Q, Liu Z. Mesoporous silica nanoparticles for stimuliresponsive controlled drug delivery: advances, challenges, and outlook. Int J Nanomed. 2017;12:87-110.

19. Narayan R, Nayak UY, Raichur AM, Garg S. Mesoporous silica nanoparticles: a comprehensive review on synthesis and recent advances. Pharmaceutics. 2018;10(3):118.

20. Yang X, Li Y, Liu X, Huang Q, Zhang R, Feng Q. Incorporation of silica nanoparticles to PLGA electrospun fibers for osteogenic differentiation of human osteoblast-like cells. Regen Biomater. 2018;5(4):229-238.

21. Shi M, Zhou Y, Shao J, et al. Stimulation of osteogenesis and angiogenesis of hBMSCs by delivering $\mathrm{Si}$ ions and functional drug from mesoporous silica nanospheres. Acta Biomater. 2015;21:178-189.

22. Song B, Wu C, Chang J. Dual drug release from electrospun poly(lacticco-glycolic acid)/mesoporous silica nanoparticles composite mats with distinct release profiles. Acta Biomater. 2012;8(5):1901-1907.

23. Dorati R, Detrizio A, Modena T, et al. Biodegradable scaffolds for bone regeneration combined with drug-delivery systems in osteomyelitis therapy. Pharmaceuticals. 2017;10(4):E96.

24. Curry AS, Pensa NW, Barlow AM, Bellis SL. Taking cues from the extracellular matrix to design bone-mimetic regenerative scaffolds. Matrix Biol. 2016;52-54:397-412.

25. Sehgal RR, Carvalho E, Banerjee R. Mechanically stiff, zinc crosslinked nanocomposite scaffolds with improved osteostimulation and antibacterial properties. ACS Appl Mater Interfaces. 2016;8(22): 13735-13747.

26. Oliver WC, Pharr GM. An improved technique for determining hardness and elastic modulus using load and displacement sensing indentation experiments. J Mater Res. 1992;7(6):1564-1583.

27. Liu ZS, Li WK, Huang CY. Synthesis of mesoporous silica materials from municipal solid waste incinerator bottom ash. Waste Manag. 2014; 34(5):893-900.

28. Wang B, Zhou Y, Li L, Wang Y. Preparation of amidoxime-functionalized mesoporous silica nanospheres (ami-MSN) from coal fly ash for the removal of U(VI). Sci Total Environ. 2018;626:219-227.

29. Trinca RB, Westin CB, da Silva JAF, Maria MÂ. Electrospun multilayer chitosan scaffolds as potential wound dressings for skin lesions. Eur Polym J. 2017;88:161-170.

30. Kim NR, Park HC, Kim I, Lim BS, Yang HC. In vitro cytocompatibility of $\mathrm{N}$-acetylcysteine-supplemented dentin bonding agents. $J$ Endod. 2010;36(11):1844-1850.

31. Zheng J, Lou JR, Zhang XX, et al. N-Acetylcysteine interacts with copper to generate hydrogen peroxide and selectively induce cancer cell death. Cancer Lett. 2010;298(2):186-194.

32. Tse HN, Tseng CZ. Update on the pathological processes, molecular biology, and clinical utility of $\mathrm{N}$-acetylcysteine in chronic obstructive pulmonary disease. Int J Chron Obstruct Pulmon Dis. 2014;9:825-836.

33. Jun JH, Lee SH, Kwak HB, et al. N-acetylcysteine stimulates osteoblastic differentiation of mouse calvarial cells. J Cell Biochem. 2008; 103(4):1246-1255.

34. Xue J, Xie J, Liu W, Xia Y. Electrospun nanofibers: new concepts, materials, and applications. Acc Chem Res. 2017;50(8):1976-1987. 
35. Mehrasa M, Asadollahi MA, Nasri-Nasrabadi B, et al. Incorporation of mesoporous silica nanoparticles into random electrospun PLGA and PLGA/gelatin nanofibrous scaffolds enhances mechanical and cell proliferation properties. Mater Sci Eng C Mater Biol Appl. 2016; $66: 25-32$.

36. Mehrasa M, Asadollahi MA, Ghaedi K, Salehi H, Arpanaei A. Electrospun aligned PLGA and PLGA/gelatin nanofibers embedded with silica nanoparticles for tissue engineering. Int J Biol Macromol. 2015; 79:687-695.
37. Zheng F, Wang S, Wen S, Shen M, Zhu M, Shi X. Characterization and antibacterial activity of amoxicillin-loaded electrospun nanohydroxyapatite/poly(lactic-co-glycolic acid) composite nanofibers. Biomaterials. 2013;34(4):1402-1412.

38. Xie J, Peng C, Zhao Q, et al. Osteogenic differentiation and bone regeneration of iPSC-MSCs supported by a biomimetic nanofibrous scaffold. Acta Biomater. 2016;29:365-379.

\section{Publish your work in this journal}

The International Journal of Nanomedicine is an international, peerreviewed journal focusing on the application of nanotechnology in diagnostics, therapeutics, and drug delivery systems throughout the biomedical field. This journal is indexed on PubMed Central, MedLine, CAS, SciSearch $®$, Current Contents ${ }^{\circledR} /$ Clinical Medicine,
Journal Citation Reports/Science Edition, EMBase, Scopus and the Elsevier Bibliographic databases. The manuscript management system is completely online and includes a very quick and fair peer-review system, which is all easy to use. Visit http://www.dovepress.com/ testimonials.php to read real quotes from published authors. 